\title{
Towards a smart toy ecosystem for pets
}

\author{
Van-Tien Hoang, Lucas Hof \\ Department of Mechanical Engineering \\ École de technologie supérieure \\ Quebec, Canada \\ van-tien.hoang.1@ens.etsmtl.ca \\ lucas.hof@etsmtl.ca
}

\begin{abstract}
When pet's owners take care of their pets, it sparks the need to know about the well-being of their pets. This requires the ability to monitor the animals' activities in order to recognize unexpected behavior of the pets. Such monitoring systems are also useful for pet training purposes, which could be extended to more intelligent systems used to encourage specific behavior of pets. In order to do so, this study aims to develop a system that can keep track and record of such data of pets (e.g. movements, body temperature). The proposed eco-system includes a collar affixed to the pet, interacting with a mobile application, a cloud data storage and processing platform as well as other toys to communicate with. This system is designed to be easily extended with new toys. It also can interact with smart voice assistant systems such as Apple Siri or Amazon Alexa. In addition, it has light weight so it can be affixed to small dogs or cats.
\end{abstract}

Keywords-Internet-of-Things (IoT); software architecture; pets; smart systems; pet behavior classification; mobile applications; cloud computing

\section{INTRODUCTION}

Human has started living with pets for thousands of years [1]. That long history of relationship between human and pets create strong bonds between them ( [2, 3]). That could be one of the reasons why there are more than 400 million domestic dogs world wide [4]. Particularly, in the United States of America, there are more than 180 million dogs and cats in 2017 [5].This results in a huge expenditure for pets, e.g. more than 69 billion dollars spending on foods, medical and other care (see more [6]). In particularly, the market sales of smart pet products is $\$ 565$ million in 2018 , which is up $11 \%$ from 2017 [7].

When the owners take care of their pets, it leads to the need to know about their well-being. There is an ongoing trend that pet-owners want systems to monitor their pet's activities and to recognize their behavior patterns. These system must be able to detect abnormal activities to alert and support the owner to take corrective measures [8, 9].

\section{A. Review of related work and products}

Currently, there are several works related to smart-systems for monitoring and behavior recognition. These can be categorized as commercial products: 1) available in the market and 2) in test or research phases.

1) Commercial products: An example of a commercialized product is Mookkie [10], a smart bowl identifying each pet visually ("face ID") and associate them with specific food. As a result, the diet can be customized for each pet. The system comes with a dedicate smartphone application providing notifications and short videos to the owners. However, this smart bowl does not interact with other "Mookkie" bowls or with other connected devices in the house.

Another product that helps the owner to achieve optimal pet diets is Sure Petcare's Pet Feeder Connect [11]. This product uses a scale to create a specific portion of food for pets. It also monitors the pets' food consumption behavior. This system identifies each pet using a wearable tag for each animal. The feeder connects to a 'hub' via a low energy protocol. The hub then uses a WIFI connection to communicate with the mobile phone, which in turn connects to the internet.

Petpace [12] is also a product to monitor the pets' wellbeing. It consists of a collar monitoring dog's vital data and alerts the owner when abnormality happens. Its architecture consists of three layers. The first layer contains the smart sensors affixed to the dog's collar. This sensor sends the actual data to the middle layer which is a base station. The base station communicates with the sensors and combines the data to send them to the cloud layer using the standard Ethernet interface. The calculation and analysis happen at the cloud layer. The owner can accesses those data wirelessly using their mobile application to consult their dog's Health Index, Activity Index, and Burned Calories.

Similar to Petpace, Fit bark [13] is a dog activity and sleep monitor device. It uses Bluetooth Low Energy (BLE) to make the connection with a WiFi base station. This technology promises lower energy consumption during data transmission, extending the lifetime of the battery in the collar. Any Android or iOS operated smartphone can be used as base station.

Another device, Scollar [14], is a dog tracking solution that provides information about the dog's activity and which offers tracking functions. This system contains a base station (e.g. a smartphone) including a WiFi connection. Data collection and transfer is the same as in the previous devices. The main difference is that Scollar is the only device that provides a GPS sensor and dog tracking service. The collar also contains a temperature sensor and integrated battery, with an estimated lifetime of 45 up to 60 days.

2) Research studies: In addition to these commercialized products, there are multiple academic studies about wearable devices for animals. For example, Pet Buddy [15] is a device for dogs which aims to monitor physical behavior 
of the pets. The device is based on an Arduino mainboard, including gyroscope sensors. The collected sensor data are later transferred via Bluetooth to another Arduino-based logic board, which itself is connected to a computer to analyze the canine behaviors. Similar to Pet Buddy, WagTag [16] is another solution to track dogs' activities which uses Bluetooth to transfer data to the computer.

Instead of monitoring basic physical activities, David Sec et. al [17] propose a system to monitor health condition of dogs to detect possible epileptic seizures. This wearable device has a noise sensor to identify respiratory frequency and it includes a heart beat sensor. It exchanges data via BLE 4.0 with a Raspberry running a Windows IoT operating system. The collected data are sent to a processing server via file transfer protocol (FTP).

The similarities of the three aforementioned works are the isolation of the wearable devices: they are not designed to be integrated with external services or operate in a network with other connected IoT devices.

One example which demonstrates the possible interactions between multiple devices is from Y. Guo et. al [18]. They prototype a wireless sensor platform for livestock (i.e., cows) behavior tracking. The board has a GPS receiver and temperature, magnetometer, and accelerometer sensors. The nearby animals of the herd form a wireless sensor network to relay the data to the central server. However, due to the integration of multiple sensors and functionalities, the device is too heavy for domestic pets. Also, domestic pets are not constantly near each other to form such a wireless sensor network.

3) Related products designed for humans: Besides aforementioned solutions, there are other devices close to the project. These products inspire us to develop the collar and the mobile application's functionalities.

- Oblu [19]. Oblu is a universal sensing platform for IoT using BLE 4.1 for communication. This development kit contains sensors such as an accelerometer, a gyroscope, and GPS, and it is a potential candidate to build our wearable device.

- Flora [20]. The Flora board is a small (1.75" diameter, 4.4 grams) wearable electronic platform. It is a typical representation of many Arduino compatible devices. This board only contains a micro-controller and programming circuits, without using any batteries and sensors. Flora is also a possible candidate for our purposes.

- GENEActiv Wireless [21]. This company offers four variations of bracelets (wristband) for humans. Depending on the variant, it includes an accelerometer, body temperature sensor, skin temperature sensor and a gyroscope, only the wireless version is equipped with live wireless data transmission via a Bluetooth interface. The built-in three-axis gyroscope allows readings up to $1 \mathrm{kHz}$. The battery life ranges from 8 hours (wireless version) to 45 days (original version).

- MbientLab MetaWear [9]. This is a smart sensor module for motion tracking and contains an accelerometer, gyroscope, pressure, thermometer sensor, and special mathematical preprocessor to combine data from each sensor integrated on the board. This device can be paired with an BLUE gateway hub to transmit data to the cloud.

- Fitbit [22] is another human activities' tracker. It is small (e.g. wrist or pocket size) and contains various sensors to track steps and estimate energy consumption, sleep and movement actions. The battery life is around 3 days and the device can store accumulated data of the time span of 1 week. The Fitbit device transfers data wireless to a base station which is also a charging device. This device is a great example of popular wearable devices in the market.

- Xiaomi MiBand [23]. This is the fitness tracker for humans. It contains an accelerometer and notification LEDs. With the original manufacturer's firmware, this device only provides aggregated data. The battery can last up to 30 days.

\section{B. Proposed project}

Although smart pet systems with the targeted specifications and objectives start to appear on the market, they are often mono-function stand-alone devices such as a single health monitor, activities tracker or feed dispenser. They are also mostly heavy and require strong power supply sources such as big battery packs or power lines. Existing systems are not designed to connect and control other smart toys for pets. In addition, they cannot be integrated into existing smart voice assistant system such as Google Home or Apple Siri. Hence, users still end up having multiple systems to handle, which is undesirably hindering from gain interest for such systems among pet-owners.

The proposed study aims to contribute to overcome these issues and offer multi-use smart products for pets (owners).

In this study, we propose an ecosystem for pets' toys consisting of a collar, toys, and a mobile application to communicate with the collar.

The proposed collar is designed to contain/sense ambient temperature, magnet field, acceleration, BLE and a speaker.

Currently, the collar prototype does not have as many sensors and functionalities as the aforementioned devices, but we will gradually improve its design and application.

Our proposed software is fed by collected data from a collar for monitoring purposes. These data are motion, ambient temperature, and magnetic proximity. The motion data are gathered from the acelerometer sensor, while proximity data between the collar and designated objects are measured via a magnetic field sensor. The environment temperature is transmitted by an ambient sensor. All those sensors transmit data via BLE to the receiver (e.g., mobile phone application). This information could also be used to encourage specific behavior of pets for training purposes. For example, to prevent the pet, (e.g. a dog), to jump inside the living room, the owner can activate an external device (e.g. a food dispenser machine to provide foods) whenever the system detects that the dog is jumping, which distracts the pet. To fulfill these objectives, it is needed to develop a system that can keep track and record data of pets (e.g. movements,). 
Based on collaboration with $\mathrm{BeOneBreed} \mathrm{-} \mathrm{a} \mathrm{local} \mathrm{industrial}$ partner - who possesses extensive expertise in making toys for pet, we are developing a prototype system to interact with small pets such as cats or dogs. The proposed system includes a collar attached to a pet, which interacts with other peripheral devices such as a food dispenser, interactive moving toys, etc. An early stage prototype of the collar was provided by BeOneBreed.

\section{Project's objectives}

The project aims to create an ecosystem of connectivity products (IoT) for pets. This ecosystem can include a vast range of product types from feeding, tracking, health monitoring to interactive toys for animals.

A key aspect of this main objective is to create a system of smart devices for pets, which integrates with existing smart home systems. It will provide the software layer for communicating and exchanging data as well as sending control commands between many smart home objects and systems such as Google Nest or Amazon Alexa. This software layer acts like a proxy to translate high level commands to BLE commands to access raw data on the collar. It also convert raw sensor data to common formats such as CSV or JSON. For example, the pet's owner asks Alexa: "what is my dog doing?". Alexa translate that sentence to a specific command set and sends to the BLE hub (or mobile application) which acts as the middle sofware layer. The BLE hub translates again that high level commands to BLE commands to get the collar data to provide the predictions. Then it provides the high level answer back to Alexa which in turn speaks to the owner.

We aim to develop an integrated ecosystem of pets' smart products including a collar and a wide range of connectivity devices (e.g., toys) focusing on the following key features:

- The wearable items are robust and lightweight.

- The wearable items have low power consumption.

- The wearable device must be able to connect to multiple devices (e. g., 'smart toys') in real-time.

- The communication between the wearable and the nonwearable devices must be fast, and reliable.

- The time windows of data exchange between devices must be minimized because of the non-stationary nature of the pets. The size of data also must be minimized (e.g., compressed).

- These products can communicate with each other and with current smart home systems. Such systems often include a collar attached to the pets, and several connected entities such as a food dispenser, and other toys.

At the same time, the project also aims to improve humananimal interaction by understanding their pets' behaviors via connectivity products. The data are collected from a portable device attached the pets and other connected devices installed inside a domestic environment. Based on those data, the system allows pet owners to decide best actions to take according to the pet's behaviour. In order to do that, the proposed mobile application collects and displays pet motion behaviors to inform owners about abnormal motion patterns of their pets. The details of this step are described in Section III-D of the System Design and Discussion part (III).

Our proposed system will include:

- A range of communicative products allowing better understanding of the animal's behavior, to assist identification of their habits enabling a medical and/or behavioral diagnosis. Thus, the system detects and records the events and activities of the animal.

- A range of communicative products assisting to monitor pets' behavior to optimize care by creating interactions. The system has to be interactive and trigger remotely programmed events.

- A range of communicative products which are easy to use, either for installation or handling (application software must be user-friendly).

In addition to the main objectives, specific interactions between the products are essential to the success of the project:

- Direct interaction between collar and accessories;

- Possibility to have multiple collars in the same area and identification of each of them;

- Direct interaction between accessories and the user (via an app) provided by wireless technology;

- No direct interaction between accessories and internet;

- Interaction with internet only from the user (via an app);

- User-specific configurable functions (via an app);

- Ability to add an interaction between the accessories.

On the other hand, each of the communicative products also has specific features. The function can allows us to propose a modular technical approach, with the development of three electronic modules, either an electronic module specific to the collar, a generic module for the ephemeral objects, and a module specific for each of the peripheral objects.

\section{Methodology}

We define the following principles for system development based on the projects' objectives:

- Optimize the software for wearable devices: based on the hard- and software specifications of the device, create 1) the core software interface (i.e. APIs) to communicate with the device via Bluetooth from the mobile application, 2) develop the control commands for the mobile application with functionalities such as reading, writing, storing data periodically from the connected collar to the application, then 3) create the reporting functions based on collected data.

- Minimize energy and resources consumption at a local device by moving all data to the cloud for heavy processing: including 1) design the software architecture and technologies to coordinate the cloud computing and the mobile application, 2) develop the ability to sync (e.g. downloading and uploading) data to the cloud from the mobile application 3) develop the interface for data management functionalities on the cloud server.

- Maximize the integration and expansion abilities of the system: based on the requirements, 1) propose a possible 
IoT gateway to communicate with connected collars, 2) propose the functionalities of the gateway; the gateway must be able to connect with multiple collars and other future 'smart toys' of BeOneBreed, and 3) propose the solution to connect the IoT gateway to an existing smart home controller service like Google Home to command the device.

\section{SYSTEM DESIGN AND DISCUSSION}

The main goal is to create an ecosystem for smart toys. It has the collar which plays a central role for communication and data collections as well as dashboard display. Furthermore, it is possible to have a BLE hub to WiFi gateway which can be used when there is no mobile application available. The hub has the similar functions as the mobile application. Figure 1 shows an overview of the entire ecosystem.

\section{A. Proposed system architecture}

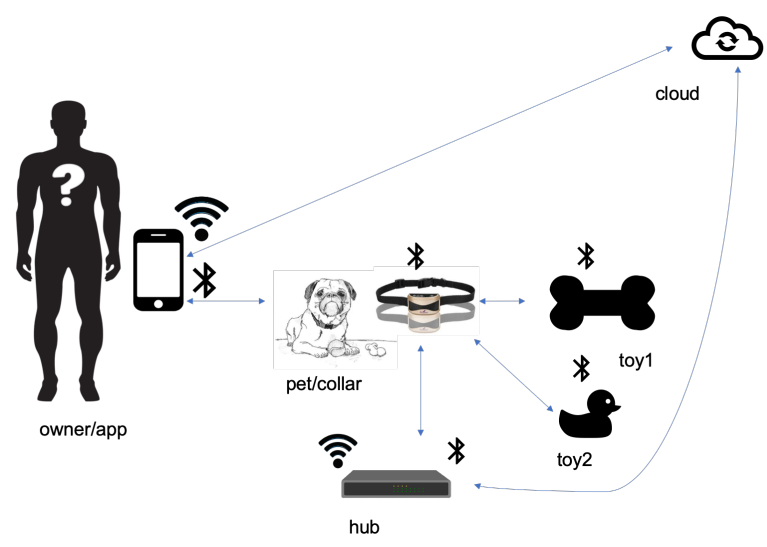

Fig. 1. Overview of the ecosystem and its components.

Based on the abstraction level of the ecosystem, we proposed an architecture, including both software and hardware components for the project, as outlined in Figure 2 .

The overview of each component and its functionalities is detailed below.

- The toy: is a Bluetooth connected device, which communicates directly with the collar and the hub. It sends the data and can be triggered by the collar.

- The mobile application: is the main user control application. The pet owner uses it to see information about their pet's activities. The main functions of the application are to trigger commands in the collar and send/retrieve data from the cloud server.

- The cloud: is the main data storage and data processing unit. After having historical data from the pet's behaviors, the analyzing procedures are executed in the cloud to perform activity prediction and other data processing. The results are transferred to the mobile application for presentation to end users.

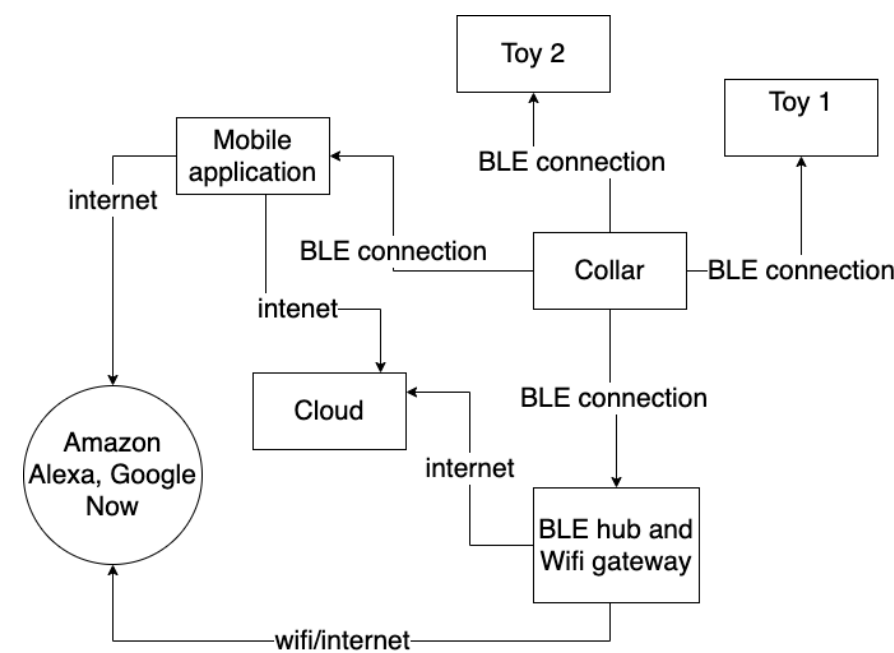

Fig. 2. Abstracted view of the system architecture. The arrows indicate the data connection between components.

- BLE hub and WiFi gateway: when the users do not have the mobile application to collect data, this gateway will handle this part and send all those data to the cloud. Moreover, it is the connection point for smart voice assistant systems to control the entire smart toy ecosystem.

\section{B. Early collar prototype}

The collar is the centre of the system. BeOneBreed provided a concept prototype that can be used. This preliminary collar prototype uses the nRF52832 ARM Cortex-M4F in form of the BC832 miniature module from Fanstel 3. The outer case components are produced by a 3-D printer. The collar features a wireless charging battery pack, its weight is about 50 gram with standard configuration and it has 3 types of sensors: an accelerometer sensor, a magnet field sensor, and an ambient temperature sensor. The collar communicates via BLE 5.0 and has a firmware developed by Motsa 1 .

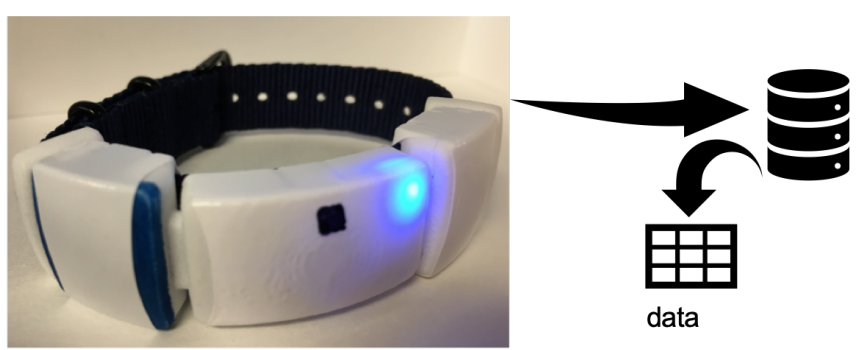

Fig. 3. The preliminary prototype collar as designed by Motsai. Currently, only raw sensor data is stored.

\section{Proposed mobile application}

As a starting point, we develop a mobile application connecting with the collar to provide the most crucial functions:

${ }^{1}$ http://motsai.ca 
- The application collects the data from the sensors in realtime when in range.

- The application provides the graphs and aggregated statistic information from the data.

- The application will be able to run on iOS or Android based phones.

- The application can store and synchronize data with the cloud database.

- Extended functions: can control the collar via digital voice assistance.

Figure 4 outlines a sketch of the mobile application with its core functions and hardware and data requirements. It features three key components: the collar, the cloud and the mobile application.

The first component is the collar. It collects the data from the sensors. For example, the accelerometer data show the moving speed of the pets and the temperature sensor retrieves the relatively skin thermal level of the pets. These data can be sent to the application in real-time or in small quantity.

The second component is the cloud. This is the place where long-term data are stored and computation of intensive tasks is executed. For example, prediction of abnormal patterns of pets based on monthly activities using deep learning.

The third part is the mobile application. Its main goals are to interact with the user. It acquires data directly from the collar via Bluetooth protocols and it receives data from the cloud for analysis. The application sends its data to the cloud.

To realize the mobile application functionalities, it is needed to feed the data from the other components. For example, to be able to display pet's behaviour statistics, the mobile software parts need to access the historical data from the cloud. Those data are collected by the collar from the built-in sensors. The processing unit at the cloud is key: it uses algorithms to predict what activities the pet has performed. The mobile application displays the analytic results in an expressive way to pet's owner.

All the basic functions such as download data from the collar, start and stop the data recording are implemented.

Auto-activity classification based on motion data will be added in a next step: the goal is to display a summary of daily or hourly activities of the pet by using accelerometer data.

\section{Pets' behavior classification on mobile device and collar}

- Data preparation: in this step, we collaborate with the industrial partner to collect the data. The partner has a controlled environment with a video camera and the pet inside. The camera records the motion activities of the pet while the collar collects measurements from the accelerometer sensor. By observing the pet actions, the partner creates a labelled dataset by associating the pet motion with the sensor data at the same time point. Later, we use this data for modeling in further steps.

- Behavior classification on a mobile application: we are using the aggregated data on days and hours to the classification. Despite to the limitation of training and labeling data, the supervised machine learning method is used to classify the motion intensity and assign them as: stationary (e.g. sleeping, resting), moderate movement (e.g. normal walking inside the house) and fast moving (e.g. running). This procedure is performed automatically without user intervention. In particularly, we are experimenting standard machine learning algorithm such as linear regression [24], random forest classifier [25], suport-vectors machine [26] and deep neural network (e.g, LSTM) [27]. For current data, it shows that random forest performs better than the others.

- Behavior classification on the collar: the previous approach has some limitations such as requiring relatively big storage memory for raw sensor's data, running completely offline. To overcome such issues, we propose another embedded application to classify motion activities of the pet. This embedded application runs in real-time and produces instantly prediction about the pet's behavior at a specific time. Now, only the prediction data are stored inside the collar, which significantly reduce the memory demand. However, the classification application requires a trained model. This trained model must be produced by a supervised learning algorithm running on an external device such as computer. It also requires enough training and labeling data to be able to predict accurately.

\section{CONCLUSION AND RESUlTS}

\section{A. Current results}

We have proposed a smart toy's ecosystem for pets which is robust to change, extensible and well-integrated in existing smart home systems. It is possible to add new toys to the system, as long as the new toys are BLE compatible. We also designed a system architecture including software architecture and hardware components. With the help of the cloud, the smart collar can be controlled over general Internet via the mobile application and the hub. This can be useful for remote monitoring and detection of abnormal activities. The preliminary collar prototype is a lightweight wearable IoT device for pets and will have the ability to be programmed for complex processing in next steps. In addition, it has a mobile application capable of recording sensor data from the collar and controlling its basic functions. For better understanding our work, we provide an brief summary of steps to achieve the project goals:

1) Collect hardware requirements (done by industrial partner)

2) Collect software requirements

3) Hardware implementation (out-source)

4) Software design: system architecture design, mobile application design, BLE hub design

5) Software implementation: mobile application, cloud storage implementation, BLE hub

When implementing this project, we face some obstacles:

1) Have to iterate many round to get the suitable requirements of the hardware and software. 


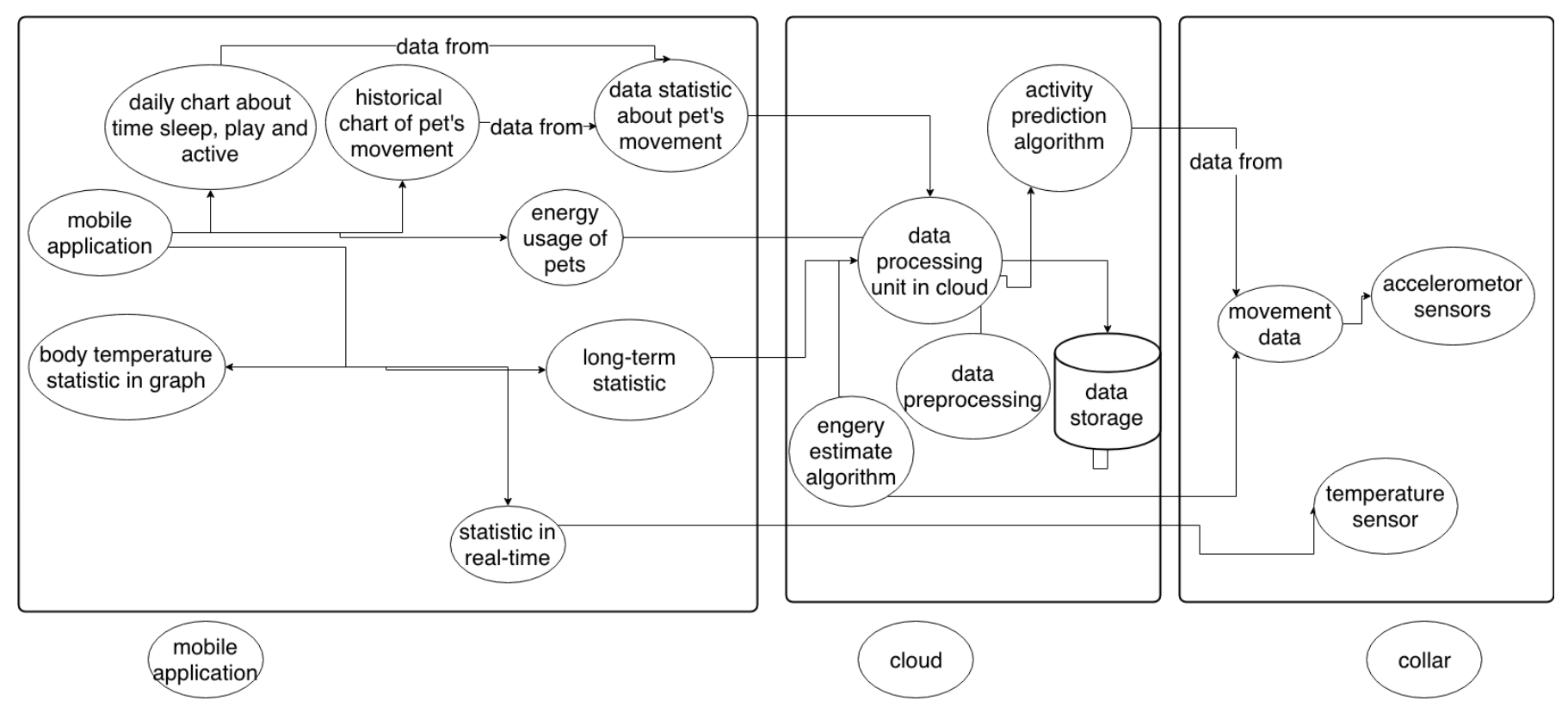

Fig. 4. The mutual connection between mobile application and other components.

2) Whenever requirements change, we have to adapt the software to its purpose. This process is not optimal but enough for a working prototype

3) The battery time is short relatively to the requirements. It can be increased by energy-optimization-ed firmware and equipping more battery cells.

\section{B. Future works}

In the next phase of the project, extended functionality of the mobile application will be developed, e.g. unexpected behavior pattern detection, based on the sensor data synchronized to the cloud. Using long-term history data of a pet and machine learning approaches, insights will be extracted to better understand the well-being of pets.

Furthermore, to increase the efficiency of data storage and battery life, a short-term behavior classification application will be developed and deployed in the collar. This embedded software will run in a real-time manner on the current sensor data to store pet motions prediction in the collar. Then it is not required to store raw sensor data, allowing the internal memory (8 MB) to last for two years. Each minute one predicated motion will be stored, consuming 20 bytes ${ }^{2}$. This results in an extended battery life from hours to weeks improving significantly the user experience of the device in the smart ecosystem.

\section{ACKNOWLEDGMENT}

The authors would like to thank the team of BeOneBreed for the fruitful discussions, their support and initiation of this project. This research is funded by a Mitacs Accelerate

\footnotetext{
${ }^{2} 20$ bytes is the minimum size of each sample

3 https://www.beonebreed.com
}

program. We also thank Motsa ${ }^{4}$ and Dr. Rolf Wuthrich for the fruitful technical discussion which helped to accelerate the project.

\section{REFERENCES}

[1] Juliet Clutton-Brock. A natural history of domesticated mammals. Cambridge University Press, 1999.

[2] James Serpell and Priscilla Barrett. The domestic dog. Cambridge University Press, 2016.

[3] Jessica Gall Myrick. Emotion regulation, procrastination, and watching cat videos online: Who watches internet cats, why, and to what effect? Computers in human behavior, 52:168-176, 2015.

[4] Yuval Noah Harari. A brief history of humankind. Publish in agreement with The Deborah Harris Agency and the Grayhawk Agency, 2014.

[5] American pet products association, inc. https://www. americanpetproducts.org (Accessed on 10/21/2019).

[6] Facts + statistics: Pet statistics.

[7] Pamela N. Danziger. Pets Are Going Digital: The Brands Pioneering The $\$ 565$ Million Market For Smart Pet Products.

[8] Pet ownership and human health: a brief review of evidence and issues.

[9] McNicholas et al. Pet ownership and human health: a brief review of evidence and issues. $B m j$, 331(7527):1252-1254, 2005.

[10] Mookkie - the pet bowl with a.i. https://www.mookkie. com/ (Accessed on 10/17/2019).

[11] Sureflap - award winning microchip cat doors \& feeders. https://www.surepetcare.com/en-US (Accessed on 10/17/2019).

${ }^{4}$ http://motsai.com 
[12] Smart dog collar - heart rate monitor \& more - petpace. https://petpace.com/. (Accessed on 10/17/2019).

[13] Fitbark 2 dog activity monitor - fitbark. https://www. fitbark.com/store/fitbark2/. (Accessed on 10/17/2019).

[14] Scollar the smart pet collar - scollar makes caring for your pets as easy as loving them. https://www.scollar. $\mathrm{com} /$. (Accessed on 10/17/2019).

[15] Juneyoung Ahn et al. Pet buddy: A wearable device for canine behavior recognition using a single imu. In 2016 International Conference on Big Data and Smart Computing (BigComp), pages 419-422. IEEE, 2016.

[16] Gary Weiss et al. Wagtag: a dog collar accessory for monitoring canine activity levels. In Proceedings of the 2013 ACM conference on Pervasive and ubiquitous computing adjunct publication, pages 405-414. ACM, 2013.

[17] David Sec et al. System for detailed monitoring of dog's vital functions. In International Conference on Computational Collective Intelligence, pages 426-435. Springer, 2018.

[18] Ying Guo et al. Animal behaviour understanding using wireless sensor networks. In Proceedings. 2006 31st IEEE Conference on Local Computer Networks, pages 607-614. IEEE, 2006.

[19] oblu iot - oblu - an open platform for wearable motion sensing. https://www.oblu.io/. (Accessed on 10/17/2019).

[20] Flora - wearable electronic platform: Arduino-compatible [v3] id: 659 - \$14.95 : Adafruit industries, unique \& fun diy electronics and kits. https://www.adafruit.com/ product/659. (Accessed on 10/17/2019).

[21] Wrist-worn accelerometer research watches - activinsights. https://www.activinsights.com/products/ geneactiv/. (Accessed on 10/17/2019).

[22] Fitbit official site for activity trackers \& more. https: //www.fitbit.com/home. (Accessed on 10/21/2019).

[23] Mi global home. https://www.mi.com/global/miband. (Accessed on 10/17/2019).

[24] Sanford Weisberg. Applied linear regression, volume 528. John Wiley \& Sons, 2005.

[25] Leo Breiman. Random forests. Machine learning, 45(1):5-32, 2001.

[26] Corinna Cortes and Vladimir Vapnik. Support-vector networks. Machine learning, 20(3):273-297, 1995.

[27] Sepp Hochreiter and Jürgen Schmidhuber. Long shortterm memory. Neural computation, 9(8):1735-1780, 1997. 